\section{Avaliação da poluição ambiental causada por particulado de chumbo emitido por uma reformadora de baterias na cidade do Rio de Janeiro, Brasil}

\author{
Assessment of environmental pollution \\ from lead particles emitted by a battery repair \\ shop in Rio de Janeiro, Brazil
}

\author{
Simone Lorena Quiterio 1 \\ Fátima Ramos Moreira 2 \\ Célia Regina Sousa da Silva 1 \\ Graciela Arbilla 1 \\ Ulisses César Araújo ${ }^{2}$ \\ Rita de Cássia O. da C. Mattos ${ }^{2}$
}

1 Instituto de Química, Universidade Federal do Rio de Janeiro, Rio de Janeiro, Brasil.

2 Escola Nacional de Saúde Pública Sergio Arouca, Fundação Oswaldo Cruz, Rio de Janeiro, Brasil.

Correspondência F. R. Moreira Centro de Estudos da Saúde do Trabalhador e Ecologia Humana, Escola Nacional de Saúde Pública Sergio Arouca, Fundação Oswaldo Cruz. Rua Leopoldo Bulhões 1480, Rio de Janeiro, $R J$ 21040-210, Brasil. fmoreira@ensp.fiocruz.br

\begin{abstract}
In this study, the lead (Pb) levels in the air surrounding a battery repair shop were measured before and after installation of an exhaust system. Samples were collected in six and four points located at approximately $25 \mathrm{~m}$ and $500 \mathrm{~m}$, respectively, from the battery repair shop. The limit of $1.5 \mathrm{mg} \mathrm{Pb.m}^{-3}$ air was exceeded in $50 \%$ of the

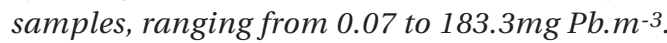
After changes in the battery repair shop's interior layout and use of the exhaust system, three points were selected, corresponding to the highest concentrations found previously. The new levels ranged from 0.8 to $17.6 \mathrm{mg} \mathrm{Pb.m}^{-3}$, demonstrating a significant decrease in the lead content discharged into the environment after meeting the exhaust requirements.
\end{abstract}

Lead; Environmental Monitoring; Environmental Pollution

\section{Introdução}

O chumbo é um elemento de ocorrência natural, encontrado em relativa abundância na crosta terrestre, quase sempre como sulfeto de chumbo, sendo que as maiores fontes geológicas do metal são as rochas ígneas e metamórficas. As suas principais fontes naturais incluem as emissões vulcânicas, o intemperismo das rochas e as emissões provenientes do mar. No entanto, devido à intensa exploração desse metal pelo homem, atualmente é difícil avaliar o conteúdo de chumbo originado dessas fontes naturais. O chumbo é o metal pesado mais profuso no ambiente 1,2,3.

As fundições primárias e secundárias de chumbo e as fábricas e reformadoras de baterias são as fontes mais significativas de emissão industrial desse metal, provocando o aumento das concentrações no solo e na poeira em áreas vizinhas 4 . As emissões industriais podem aumentar os níveis ambientais de chumbo, principalmente em áreas vizinhas a essas indústrias, pois nelas são utilizados processos e tecnologia obsoletos. Em geral, tais empresas têm instalações precárias, são ampliadas sem planejamento ou cuidados com a purificação do ar, funcionando assim como fontes de emissão de chumbo para o ambiente externo 5 .

Nos países em desenvolvimento, a indústria de baterias chumbo-ácida é a maior consumidora de chumbo, e os trabalhadores deste 
setor estão sob alto risco de intoxicação pelo metal. Enquanto que, em países desenvolvidos, este risco ocupacional tem sido estudado e regulado, pouco se conhece sobre este tipo de exposição nos países em desenvolvimento. A produção dessas baterias pode ser realizada em operações de pequena escala, utilizando tecnologia relativamente simples, tornando-se atraente para economias em desenvolvimento 6,7 .

As fontes de emissão de chumbo são representadas pelas atividades de empilhamento e encaixe das placas e solda dos terminais de chumbo. A manipulação das placas resulta no desprendimento de grandes quantidades de poeira constituída, principalmente, pelo chumbo sob a forma metálica e de óxidos; reservando-se à soldagem a emissão de fumos contendo o metal 7 .

Um elevado nível de chumbo em sangue observado em populações que residem nas proximidades de áreas industrializadas, quando comparadas com populações de áreas isoladas, reflete o impacto da poluição ambiental do chumbo ${ }^{8,9}$. Recentes estudos em diversos países têm associado a exposição ambiental ao chumbo com efeitos adversos em diferentes sistemas do organismo humano, incluindo alterações nos sistemas neurológico, hematológico, metabólico e cardiovascular 10,11. Vários pesquisadores têm avaliado os efeitos dessa exposição, particularmente em crianças e jovens, que são mais sensíveis a esse metal. Esses estudos demonstram que os efeitos adversos podem ocorrer em níveis de exposição antes considerados seguros 12,13,14,15,16,17. O monitoramento biológico e ambiental realizado em tempo adequado é uma medida objetiva, que deve ser conduzida antes da introdução de um efetivo sistema de controle da poluição.

Este estudo teve como objetivo avaliar o nível de chumbo presente na área circunvizinha a uma reformadora de baterias situada na zona norte da cidade do Rio de Janeiro, Brasil, antes e depois da adoção de medidas de controle da poluição pela empresa, utilizando o ar atmosférico como indicador no monitoramento ambiental.

\section{Experimental}

$\underline{\text { Localização e climatologia }}$

Segundo dados do Instituto Brasileiro de Geografia e Estatística (IBGE), a região estudada possui uma população de aproximadamente dez mil habitantes, quatro escolas municipais e três hospitais no raio de até $500 \mathrm{~m}$ da reformadora 18 . Nessa região, segundo a classifica- ção de Köpper, o clima é Cwa (clima meso térmico com chuvas de verão e verão quente), com uma fina poeira presente no ar todos os dias. As médias anuais de temperatura, índice pluviométrico e umidade relativa foram $23,7^{\circ} \mathrm{C}$, $1.172,9 \mathrm{Lm}^{-2}$ e $79 \%$, respectivamente, no ano de 1999, enquanto que, em 2000 , ficaram em $24,2^{\circ} \mathrm{C}$, $652 \mathrm{Lm}^{-2}$ e $75 \%$, respectivamente. A direção predominante dos ventos é a nordeste (45\%), de acordo com os dados fornecidos pela estação meteorológica localizada no Flamengo, zona sul da cidade do Rio de Janeiro 19 (Figura 1).

\section{Amostragem}

O sistema de coleta do ar atmosférico foi constituído por um separador de partículas ligado ao suporte equipado com filtro de nitro celulose Millipore (Bedford, Estados Unidos), com 55mm de diâmetro e poro de $0,8 \mathrm{~mm}$, conectado a uma bomba de médio volume com vazão de 20 Lmin-1, marca Sibata, modelo IP-20T (Tóquio, Japão), colocada sobre um tripé de $2 \mathrm{~m}$ de altura.

A escolha dos pontos de coleta obedeceu às normas estabelecidas pela Environmental Protection Agency (EPA), Estados Unidos, que se aplicam na amostragem do ar em ambientes externos, quando a fonte de emissão é estacionária 20. Desta forma, há algumas recomendações que devem ser observadas tais como altura ideal, topografia, meteorologia, dispersão e deposição do chumbo 21 .

O tempo de coleta foi de cinco horas em cada um dos seguintes pontos em relação ao centro da reformadora de baterias (Figura 2): (P1) $6 \mathrm{~m}$ a sudeste; (P2) $17 \mathrm{~m}$ a sudeste; (P3) $13 \mathrm{~m}$ a nordeste; (P4) 17,5m a nordeste; (P5) $16 \mathrm{~m}$ a sudoeste; (P6) 24m a noroeste; (P7) 500m a sudoeste; (P8) 500m a nordeste; (P9) $500 \mathrm{~m}$ a noroeste e (P10) 500m a sudeste 22 .

O monitoramento do ar ocorreu durante três meses, com um intervalo de, aproximadamente, um mês entre as medições, antes da instalação do sistema de exaustão. Desta forma, foram realizadas três amostragens no período de maio a julho de 1999, sendo que, em cada uma delas, a coleta se deu em seis pontos distintos num raio de até $25 \mathrm{~m}$ da reformadora e, a 500m, aconteceu uma única coleta em quatro pontos distintos. Após as modificações na reformadora de baterias foram realizadas mais duas amostragens (abril e setembro de 2000), nos três pontos $(3,4$ e 6$)$ onde as concentrações excederam o limite de 1,5mg Pb.m-3, estabelecido pela EPA 23 .

Paralelamente a essas avaliações, uma coleta de ar também foi efetuada numa área residencial, isenta de qualquer fonte estacionária 


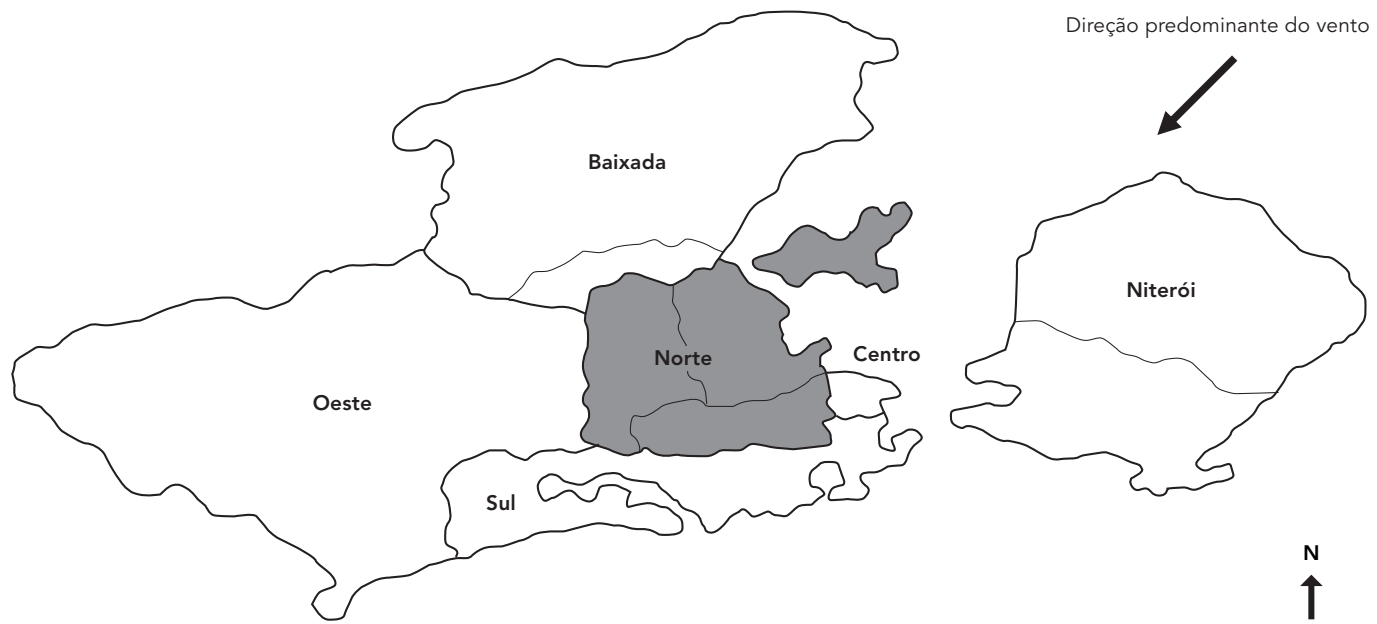

área de amostragem

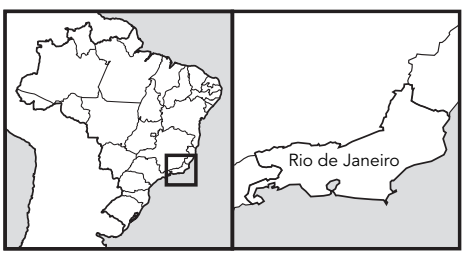

de emissão de chumbo (Recreio dos Bandeirantes, zona oeste da cidade do Rio de Janeiro) para ser utilizada como branco.

\section{Materiais e reagentes}

Todo material plástico e vidraria utilizados ficaram imersos por um período mínimo de 24 horas em solução de Extran (Merck, Elmsford, Estados Unidos) a 5\%(v/v), enxaguados abundantemente em água corrente e novamente imersos por, pelo menos, 48 horas em solução de ácido nítrico (Merck) 10\%(v/v), para descontaminação. Em seguida, foram lavados copiosamente com água ultrapura do tipo Milli$\mathrm{Q}$ (Millipore, Bedford, Estados Unidos) e secos a $40^{\circ} \mathrm{C}$ em estufa 24 .

Todos os reagentes utilizados foram, pelo menos, de grau analítico (PA). O padrão era preparado diariamente por diluições apropriadas da solução estoque de $1.000 \mathrm{mg} \mathrm{mL}^{-1}$ de chumbo (Merck) em ácido nítrico $0,2 \%$ (v/v), usandose água (resistividade $18 \mathrm{M} \Omega \mathrm{cm}$ ) previamente purificada em sistema Milli-Q.

\section{Preparação das amostras}

Os filtros foram transferidos cuidadosamente para frascos de polietileno e solubilizados em $3 \mathrm{~mL}$ de ácido nítrico concentrado à temperatura ambiente por duas horas. Após este tempo, o volume de $50 \mathrm{~mL}$ foi completado com água Milli-Q e uma alíquota diluída convenientemente para posterior leitura por ET AAS.

\section{Instrumental}

Um espectrômetro de absorção atômica modelo 5100 equipado com corretor de fundo com efeito Zeeman, forno de grafite HGA-600 e amostrador automático AS-60, todos Perkin-Elmer (Norwalk, Estados Unidos), foram utilizados neste trabalho. Tubos recobertos com grafite pirolítico (Perkin-Elmer Part N. B010-9322) e plataformas de grafite pirolítico (Perkin-Elmer Part N. B010-9324) foram usados em todos os experimentos. O comprimento de onda da lâmpada de catodo oco (Perkin-Elmer Part N. N0661299) de chumbo foi de $283,3 \mathrm{~nm}$ e a largura da 
Localização dos pontos de coleta em relação à reformadora de baterias. (P1): $6 \mathrm{~m}$ a sudeste; (P2): $17 \mathrm{~m}$ a sudeste; (P3): $13 \mathrm{~m}$ a nordeste; (P4): 17,5m a nordeste; (P5): $16 \mathrm{~m}$ a sudoeste; (P6): $24 \mathrm{~m}$ a noroeste; (P7): $500 \mathrm{~m}$ a sudoeste; (P8): $500 \mathrm{~m}$ a nordeste; (P9): $500 \mathrm{~m}$ a noroeste e (P10): $500 \mathrm{~m}$ a sudeste.

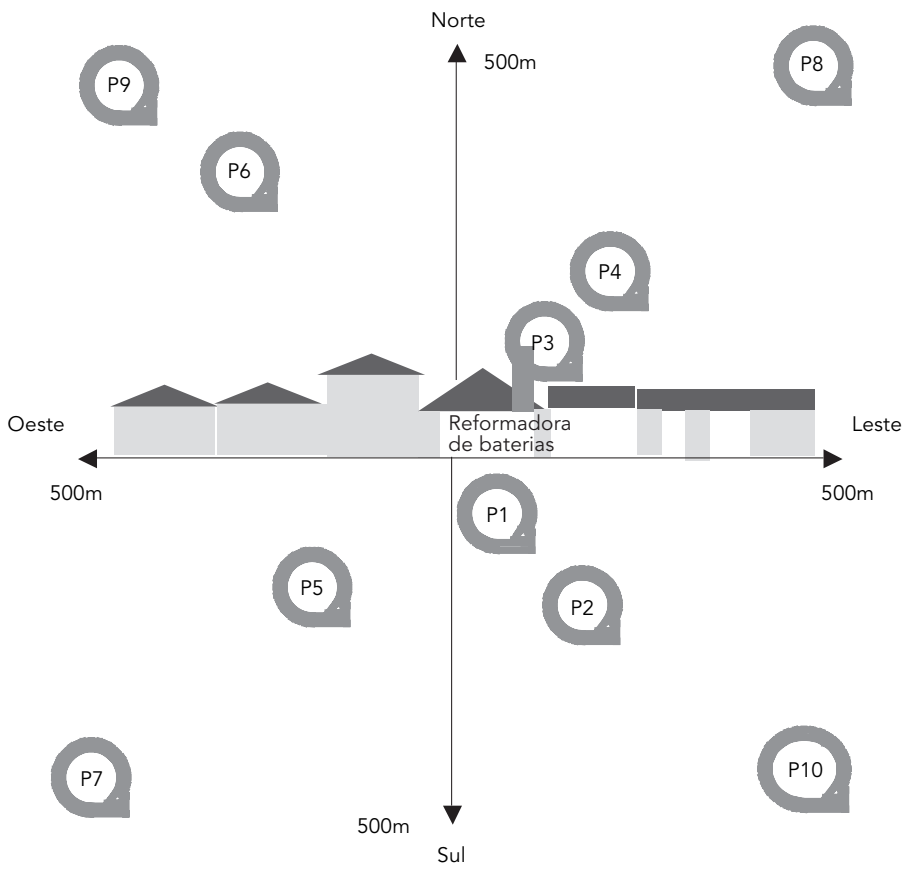

fenda foi de $0,7 \mathrm{~nm}$. A medida do sinal corrigido foi realizada em área de pico (absorvância integrada).

\section{Análise}

O programa de temperatura para a determinação de chumbo nos filtros pode ser encontrado na Tabela 1. As temperaturas de pirólise e atomização utilizadas foram $700^{\circ} \mathrm{C} \mathrm{e} 1300^{\circ} \mathrm{C}$, respectivamente, e o volume de amostra foi de $20 \mu \mathrm{L}$. As amostras foram diluídas 1+19 com água e lidas sem o uso de modificador químico.

A exatidão do método foi verificada por meio das amostras de controle interno e externo. A amostra de referência SRM 3087a (NIST, Estados Unidos - valor: 40,38 $\pm 0,92 \mathrm{mg}$ filtro- $^{-1}$ ) foi utilizada como controle interno. A concentração de chumbo encontrada para este material de referência foi $37,98 \pm 6,43 \mathrm{mg}$ filtro-1. Não houve diferença estatisticamente significativa (teste $t$ de Student, 95\% de confiança) entre os valores experimental e certificado. O controle externo foi realizado com o Instituto Nacional de Seguridad e Higiene en el Trabajo (Zaragoza, Espanha), por meio do Programa Interlaboratorial de Controle de Qualidade.

Para uma diluição de vinte vezes, o limite de detecção calculado foi de $0,3 \mathrm{mg} \cdot \mathrm{m}^{-3}$ em um filtro novo e não diluído.

\section{Resultados e discussão}

Na interpretação dos resultados encontrados para chumbo no ar, utilizou-se o valor de referência de $1,5 \mu \mathrm{g} \cdot \mathrm{m}^{-3}$, segundo as regulamentações da EPA 20.

\section{Concentração de chumbo nas amostras de ar}

A dispersão e deposição de chumbo no ambiente dependem das condições meteorológicas e dos parâmetros de emissão da fonte, ou seja, velocidade e temperatura do poluente, vazão etc., que influenciam de modo determinante na distribuição da poluição atmosférica 20 . A difusão do chumbo está amplamente relacionada com as mudanças de correntes eólicas e de temperatura existentes na camada de ar. O vento, um importante parâmetro meteorológico, é conseqüência da diferença de pressão resultante do aquecimento ou resfriamento da atmosfera pelo sol, capaz de espalhar o chumbo numa determinada região e transportá-lo para longe de seu ponto de emissão 25 .

De acordo com a Tabela 2, o chumbo se dispersa no raio de $25 \mathrm{~m}$ da reformadora de baterias, na faixa de concentração de 0,07 a 183,3mg.m-3. As maiores concentrações de chumbo foram encontradas a 13 metros na direção nordeste (média de 95,0mg.m-3), onde está localizada a principal saída de ar do setor de produção, sugerindo que as concentrações encontradas estão relacionadas com as atividades desenvolvidas na reformadora de baterias e à direção predominante do vento (nordeste). O teor encontrado para a amostra considerada como branco foi de $0,07 \mu \mathrm{g} . \mathrm{m}^{-3}$, o que é muito inferior ao limite estabelecido pela EPA (1,5mg.m-3). Assim, dos pontos amostrados, $100 \%$ das amostras coletadas em P3 e 67\% daquelas de P4 e P6 excederam este valor. Em 500m (P7 a P10), as concentrações foram equivalentes às do branco (média de 0,05mg.m-3).

Na Tabela 2, pode-se observar que, para um mesmo ponto, houve uma diferença muito grande entre as concentrações nos três monitoramentos devido aos elevados desvios encontrados. Este fato mostra o grau de impor- 
tância da atividade desenvolvida no interior da reformadora e também o papel desempenhado pelas condições climáticas no momento da avaliação.

\section{Concentração de chumbo após a instalação do sistema de exaustão}

Diversas modificações na produção tais como a reunião das atividades envolvendo a manipulação do metal em um único recinto e a manutenção do piso úmido, e melhorias (ventilação do ambiente e o uso de duto e coifa próximos e direcionados aos fumos e particulados gerados pelas atividades) na reformadora foram propostas para que houvesse uma redução na emissão do metal. Dentre estas, encontra-se a recomendação para a instalação de um sistema de exaustão. Assim, foi possível avaliar a eficiência da exaustão através do monitoramento do ar enviado para o ambiente. A seleção dos pontos recaiu sobre aqueles três que correspondiam aos mais críticos encontrados nas avaliações anteriores: P3, saída da chaminé; $\mathrm{P} 4$, varanda de residência (fundos da reformadora de baterias) e P6, galpão (à direita da reformadora de baterias), a saber, $13 \mathrm{~m}$ a nordeste; $17,5 \mathrm{~m}$ a nordeste e $24 \mathrm{~m}$ a noroeste, respectivamente.

Os resultados correspondentes à média de três monitoramentos antes da instalação do sistema de exaustão (período de maio a julho de 1999) e aqueles referentes à média das duas avaliações após as mudanças na reformadora (abril e setembro de 2000) são apresentados na Tabela 3.

Esses resultados demonstram que, após a instalação do sistema de exaustão, houve uma significativa redução dos níveis de chumbo emitidos para o ambiente. Nos pontos 4 e 6, a redução foi de 50 a 73\%, o que possibilitou a obten- ção de níveis de $\mathrm{Pb}$, em pelo menos, um dos monitoramentos, menores do que o valor de referência de $1,5 \mathrm{mg} \cdot \mathrm{m}^{-3}\left(\mathrm{P} 4=0,48 \mathrm{mg} \cdot \mathrm{m}^{-3}\right.$ e P6 = 1,4mg.m-3). Entretanto, a concentração no ponto 3 , que avalia diretamente o ar tratado pelo sistema de exaustão, mesmo com uma redução de mais de $84 \%$, ainda é aproximadamente dez vezes maior do que o limite estabelecido pela EPA.

As medições realizadas após a instalação do sistema de exaustão também apresentaram desvios que variaram de 9 (P4) a 30\% (P6) do valor das concentrações, mostrando a necessidade de se conhecer e tentar padronizar as diversas variáveis envolvidas.

\section{Conclusões}

Nessas avaliações, as concentrações de chumbo encontradas no ar ao redor da reformadora de baterias estão relacionadas à direção do vento, às saídas de ar da reformadora de baterias e

\section{Tabela 1}

Programa de Temperatura para a determinação de chumbo em filtros por ET AAS.

\begin{tabular}{lrrrr}
\hline Etapa & \multicolumn{2}{c}{ Temperatura $\left({ }^{\circ} \mathrm{C}\right)$} & Tempo (s) & Fluxo (mL.min-1) \\
\hline Rampa & "Hold" & & \\
\hline $1^{*}$ & 90 & 1 & 10 & 300 \\
2 & 120 & 10 & 10 & 300 \\
3 & 700 & 5 & 15 & 300 \\
4 & 20 & 1 & 10 & 300 \\
$5^{* *}$ & 1.300 & 0 & 5 & 0 \\
6 & 2.600 & 1 & 3 & 300 \\
\hline
\end{tabular}

* Amostra $(20 \mu \mathrm{L})$;

** Leitura.

Tabela 2

Média e desvio padrão das concentrações de chumbo nas amostras de ar coletadas antes da instalação do sistema de exaustão.

\begin{tabular}{|c|c|c|c|}
\hline Pontos de coleta & $\begin{array}{l}\text { Distância da reformadora } \\
\text { de baterias }(m)\end{array}$ & Média ( $\mu \mathrm{g} \cdot \mathrm{m}-3$ ) & $\begin{array}{c}\text { Faixa de concentração } \\
\left(\mu \mathrm{g} \cdot \mathrm{m}^{-3}\right)\end{array}$ \\
\hline P3 & 13 - nordeste & $95,00 \pm 79,10$ & $30,60-183,30$ \\
\hline P4 & 17,5 - nordeste & $4,10 \pm 4,90$ & $0,48-9,70$ \\
\hline P6 & 24 - noroeste & $2,00 \pm 0,60$ & $1,40-2,60$ \\
\hline P1 & 6 - sudeste & $0,52 \pm 0,37$ & $0,07-1,10$ \\
\hline P2 & 17 - sudeste & $0,20 \pm 0,20$ & $0,07-0,42$ \\
\hline P5 & 16 - sudoeste & $0,38 \pm 0,44$ & $0,08-0,88$ \\
\hline P7-P10 & 500 & $0,05 \pm 0,02$ & $0,03-0,07$ \\
\hline
\end{tabular}


Média das concentrações de chumbo correspondentes aos monitoramentos do ar externo, antes e depois da instalação do sistema de exaustão.

\begin{tabular}{|c|c|c|}
\hline \multirow[t]{2}{*}{ Local } & \multicolumn{2}{|c|}{ Resultados $\left(\mu \mathrm{g} \cdot \mathrm{m}^{-3}\right)$} \\
\hline & $\begin{array}{l}\text { Antes do sistema de exaustão } \\
\text { (maio a julho de 1999)* }\end{array}$ & 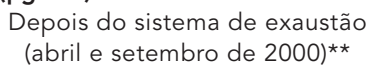 \\
\hline P3 (saída da chaminé) & $95,00 \pm 79,10$ & $15,00 \pm 4,00$ \\
\hline $\begin{array}{l}\text { P4 (varanda de residência - } \\
\text { fundos da reformadora de baterias) }\end{array}$ & $4,10 \pm 4,90$ & $1,10 \pm 0,10$ \\
\hline $\begin{array}{l}\text { P6 (galpão à direita da reformadora } \\
\text { de baterias) }\end{array}$ & $2,00 \pm 0,60$ & $1,00 \pm 0,30$ \\
\hline
\end{tabular}

$\star \mathrm{n}=3$;

$\star \star \mathrm{n}=2$.

às atividades desenvolvidas durante o período da coleta, sendo estes fatores primordiais ao planejamento da amostragem. A distância de até $25 \mathrm{~m}$ se mostrou satisfatória para coleta de chumbo no ar atmosférico para fontes fugitivas, pois os valores encontrados estão relacionados com fatores citados acima.

A instalação do sistema de exaustão, as modificações no processo de produção e as melhorias das instalações promoveram uma significativa redução dos níveis de chumbo emitidos para o ambiente. Quanto ao P3, onde a concentração ainda está acima do valor limite estabelecido pela EPA, será necessário realizar outras modificações e posterior monitoramento, a fim de verificar a eficiência das novas mudanças.

Como a concentração do chumbo no ar atmosférico nas circunvizinhanças da reforma-

\section{Resumo}

Neste estudo, determinou-se o teor de chumbo $(\mathrm{Pb})$ presente no ar ao redor de uma reformadora de baterias, antes e após da instalação do sistema de exaustão. $\mathrm{Na}$ área externa à reformadora de baterias, as amostras foram coletadas em seis e quatro pontos localizados a aproximadamente $25 \mathrm{~m}$ e $500 \mathrm{~m}$, respectivamente, da reformadora de baterias. Os resultados obtidos mostram que o limite para $\mathrm{Pb}$ no ar atmosférico de $1,5 \mu \mathrm{g}$ Pb. $\mathrm{m}^{-3}$ foi excedido em $50 \%$ nas amostras coletadas,

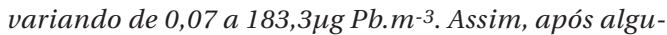
mas modificações na distribuição das atividades no interior da reformadora de baterias e a colocação do sistema de exaustão foram selecionados três pontos de coleta, que correspondiam àqueles mais críticos encontrados nas avaliações anteriores do ar externo. As no-

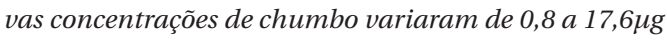
Pb. $\mathrm{m}^{-3}$, demonstrando que houve uma significativa redução dos níveis de chumbo emitidos para o ambiente após a instalação do sistema de exaustão. dora de baterias retrata o chumbo disperso na atmosfera, este continua sendo um importante agente químico a ser investigado em áreas de risco, devido aos seus efeitos adversos à saúde dos trabalhadores e residentes desses locais, e por ser considerado um problema de saúde pública internacional.

Os resultados encontrados aqui servem de orientação para a implantação de ações e conscientização dos empresários quanto à adoção de um sistema de exaustão eficiente, que ajude na redução da poluição ambiental. Acredita-se que a continuidade desse trabalho contribuirá com informações sobre a contaminação ambiental pelo chumbo para que as autoridades e os responsáveis pelas empresas executem mudanças radicais, que favoreçam a saúde dos trabalhadores e da população em geral.

\section{Colaboradores}

Todos os autores participaram no desenvolvimento do projeto. S. L. Quiterio foi responsável pela coleta e análise das amostras e colaborou na elaboração do artigo. F. R. Moreira foi responsável pela determinação da concentração de chumbo nas amostras e elaboração do artigo. G. Arbilla colaborou na elaboração do artigo. U. C. Araújo colaborou na coleta das amostras.

\section{Agradecimentos}

Os autores agradecem ao Conselho Nacional de Desenvolvimento Científico e Tecnológico e ao Centro de Estudos da Saúde do Trabalhador e Ecologia Humana, Escola Nacional de Saúde Pública Sergio Arouca, Fundação Oswaldo Cruz pelo apoio recebido para a realização deste trabalho. 


\section{Referências}

1. Baird C. Environmental chemistry. New York: W. H. Freeman and Co.; 1995.

2. Azevedo FA, Chasin AAM. Metais: gerenciamento da toxicidade. São Paulo: Editora Atheneu; 2003.

3. Stokinger HE. The metals. In: Clayton GD, Clayton FE, editors. Patty's industrial hygiene and toxicology. v. 2. New York: John Wiley \& Sons; 1981. p. 1687-725.

4. World Health Organization. Report on the Human Exposure Assessment Location (HEAL) programme meeting. Human exposure assessment series - human exposure to lead. Geneva: World Health Organization; 1992.

5. Tanner RL. Measurements in support of air quality improvement: some historical insights. Atm Environ 2003; 37:1271-6.

6. Matte TD, Figueroa JP, Ostrowski S, Burr G, Jackson-Hunt L, Keenlyside RA, et al. Lead poisoning among household members exposed to lead-acid battery repair shops in Kingston, Jamaica. Int J Epidemiol 1989; 18:874-81.

7. Araújo UC, Pivetta FR, Moreira JC. Avaliação da exposição ocupacional ao chumbo: proposta de uma estratégia de monitoramento para prevenção dos efeitos clínicos e subclínicos. Cad Saúde Pública 1999; 15:123-32.

8. Prpic-Majuc D, Pongracic J, Hrsak J, Pizent A. A follow-up study in a lead smelter community following the introduction of an effective pollution control system - Israel. J Med Sci 1992; 28:548-56.

9. Moreira FR, Moreira JC. A importância da análise de especiação do chumbo em plasma para a avaliação dos riscos à saúde. Quim Nova 2004; 27: 251-60.

10. Moreira FR, Moreira JC. Os efeitos do chumbo sobre o organismo humano e seu significado para a saúde. Rev Panam Salud Pública 2004; 15:119-29.

11. Goyer RA. Toxic effects of metals - Lead. In: Amdur MO, Dull J, Klaassen CD, editors. Casareh and Doull's toxicology - the basic science of poisons. $4^{\text {th }}$ Ed. New York: Pergamon Press; 1991. p. 639-46.

12. Shen X, Wu S, Yan C. Impacts of low-level lead exposure on development of children: recent studies in China. Clin Chim Acta 2001; 313:217-20.

13. US Department of Health and Human Services. Toxicological profile for lead. Atlanta: Public Health Service, Agency for Toxic Substances and Disease Registry, U.S. Department of Health and Human Services; 1999.

14. US Department of Health and Human Services, Public Health Service. Case studies in environmental medicine: lead toxicity. Atlanta: Agency for Toxic Substances and Disease Registry, U.S. Department of Health and Human Services; 1992.
15. Jacob B, Ritz B, Heinrich J, Hoelscher B, Wichmann HE. The effect of low-level blood lead on hematologic parameters in children. Environ Res 2000; 82:150-9.

16. Serwint JR, Damokosh AI, Berger OG, Chisolm Jr. JJ, Gunter EW, Jones RL, et al. No difference in iron status between children with low and moderate lead exposure. J Pediatr 1999; 135:108-10.

17. Wright RO. The role of iron therapy in childhood plumbism. Curr Opin Pediatr 1999; 11:255-8.

18. Centro de Informação Científica e Tecnológica, Fundação Oswaldo Cruz. Localização das fábricas de baterias do Município do Rio de Janeiro. Rio de Janeiro: Centro de Informação Científica e Tecnológica, Fundação Oswaldo Cruz; 1999.

19. Pinhel A. Condições climáticas da Cidade do Rio de Janeiro no ano de 1999. Rio de Janeiro: Laboratório de Meteorologia, Universidade do Estado do Rio de Janeiro; 2000.

20. U. S. Environmental Protection Agency. Guidance for siting ambient air monitors around stationary lead sources. http://www.epa.gov:80/ttnamtil/ files/ambient/criteria/reldocs/pbgde997.pdf (acessado em Mai/1998).

21. Valencia TC, Carrillo LL, Romieu I. Manual de procedimiento en la toma de muestras biológicas y ambientales para determinar niveles de plomo. Metepec: Centro Panamericano de Ecología Humana y Salud; 1995.

22. Quiterio SL, Silva CRS, Vaitsman DS, Moreira FR, Araújo UC, Mattos RCOC, et al. Uso da poeira e do ar como indicadores de contaminação ambiental em áreas circunvizinhas a uma fonte de emissão estacionária de chumbo. Cad Saúde Pública 2001; 17:501-8.

23. Quiterio SL, Silva CRS, Arbilla G, Moreira MFR, Araújo UC, Mattos RCOC, et al. Controle das emissões de chumbo particulado no entorno de uma reformadora de baterias da Cidade do Rio de Janeiro usando ar como indicador. Cad Saúde Pública 2003; 19:475-80.

24. Moreira MFR. Determinação direta de cádmio em sangue total e urina por espectrometria de absorção atômica no forno de grafite [Dissertação de Mestrado]. Rio de Janeiro: Departamento de Química, Pontifícia Universidade Católica do Rio de Janeiro; 1993.

25. Landrigan PJ, Gehlbach SH, Rosenblum BF, Shoults JM, Candelaria RM, Barthel WF, et al. Epidemic lead absorption near an ore smelter: the role of particulate lead. N Eng J Med 1975; 292:123-9.

Recebido em 08/Jul/2005

Versão final reapresentada em 11/Nov/2005

Aprovado em 10/Jan/2006 